DE

M E D I C I N A

T R O P I C A L

$\mathrm{DE}$

SÃO PAULO

JOURNAL OF THE SÃO PAULO INSTITUTE OF TROPICAL MEDICINE

(1)Kerman University of Medical Sciences, Research Center for Hydatid Disease in Iran, Kerman, Iran

(2)Kerman University of Medical Sciences, Sirjan School of Medical Sciences, Sirjan, Iran

(3)Shahroud University of Medical Sciences, School of Medicine, Shahroud, Iran

(4)Alborz University of Medical Sciences, Dietary Supplements and Probiotics Research Center, Karaj, Iran

(5) Shahid Bahonar University of Kerman, Faculty of Veterinary Medicine, Department of Food Hygiene and Public Health, Kerman, Iran

Correspondence to: Majid Fasihi Harandi Kerman University of Medical Sciences, School of Medicine, Research Center for Hydatid Disease in Iran, Kerman, 7616914155, Iran

Tel: +989131405616

Fax: +983433257478

E-mail: fasihi@kmu.ac.ir

Received: 22 January 2017

Accepted: 18 May 2017

\section{A conventional PCR for differentiating common taeniid species of dogs based on in silico microsatellite analysis}

Saeedeh Shamsaddini ${ }^{1}$, Mohammad Ali Mohammadi ${ }^{2}$, Seyed Reza Mirbadie ${ }^{3}$, Sima Rostami ${ }^{4}$, Mansoureh Dehghani ${ }^{1}$, Balal Sadeghi ${ }^{5}$, Majid Fasihi Harandi ${ }^{1}$

\section{ABSTRACT}

Canine taeniids are among the major tapeworms with remarkable medical and economic significance. Reliable diagnosis and differentiation of dog taeniids using simple and sensitive tools are of paramount importance for establishing an efficient surveillance system. Microsatellites as abundant unique tandem repeats of short DNA motifs are useful genetic markers for molecular epidemiological studies. The purpose of the present study was to find a primer pair for rapid differentiation of major tapeworms of dogs, Taenia hydatigena, $T$. multiceps, T. ovis and Echinococcus granulosus, by screening existing nucleotide data. All the mitochondrial genome records as well as non-coding ITS1 sequences of Taeniidae species were downloaded from Nucleotide database from NCBI. For prediction and analysis of potential loci of STR/SSR in ITS1 as well as mitochondrial regions, we used ChloroMitoSSRDB 2.0 and GMATo v1.2. software. Different tapeworm species were categorized according to different motif sequences and type and size of each microsatellite locus. Three primer sets were designed and tested for differentiating taeniid species and evaluated in a conventional PCR system. Four taeniid species were successfully differentiated using a primer pair in a simple conventional PCR system. We predicted 2-19 and 1-4 microsatellite loci in ITS1 and mitochondrial genome, respectively. In ITS1, 41 Di and 21 Tri motifs were found in the taeniids while the majority of the motifs in the mitochondrial genome were Tetra (89) and Tri (70). It is documented that the number and diversity of microsatellite loci is higher in nuclear ITS1 region than mostly coding mitochondrial genome.

KEYWORDS: Canine Tapeworms. Taeniidae. Microsatellite. Mitochondrial genome. Internal Transcribed Spacer. Taenia. Echinococcus.

\section{INTRODUCTION}

The cestodes of the family Taeniidae contain different species of Taenia and Echinococcus that are known to be transmitted between mammalian intermediate and definitive hosts. The most frequent Taeniidae tapeworms of ruminants include Taenia hydatigena, T. multiceps, T. ovis and Echinococcus granulosus sensu lato. All four species are perpetuated in a canine-ovine life cycle that involves dog as the main definitive host and sheep as the main intermediate host.

Cystic Echinococcosis (CE) as well as other canine taeniid infections are globally important zoonoses of human and animals and major medical, veterinary and economic losses are attributable to these taeniid infections ${ }^{1-3}$. Dogs have been found frequently infected with these tapeworm species. For E. granulosus, the prevalence ranges from $3.6 \%$ to $25.9 \%$ in Asia, Europe and Africa using molecular tools ${ }^{4}$. It has been shown that T. hydatigena is one of the most prevalent Taenia species in dogs 
and sheep in Iran, reported in 28.5-53.0\% of dogs, 9.1\% of foxes, $10 \%$ of jackals and $12.8 \%$ of sheep in different parts of the country ${ }^{5-7}$. Taenia multiceps, causing ovine cenuriasis, has been reported in 2.3 to $4.5 \%$ of sheep in Kenya ${ }^{8}$. In Iran, $3-40 \%$ of dogs have been found infected by the adult T. multiceps ${ }^{9}$. Previous studies on helminth parasites of dogs showed $7.2 \%$ and $24 \%$ were infected with $T$. ovis in the Western and Central regions of Iran, respectively ${ }^{6,10}$. Taenia ovis infection in an emerging disease of sheep in China ${ }^{11}$ and has been reported in the $3.2 \%$ of vegetable samples prepared for primates in Basel Zoo ${ }^{12}$.

The annual monetary losses due to T. hydatigena infection in livestock are estimated at about US\$ 65,000 and US\$370,000 in Ethiopia and Sardinia, respectively ${ }^{3,13}$. Annual losses of ovine cenuriasis due to T. multiceps in Iran has been estimated at about US $\$ 15,700^{14}$. Global monetary burden of Cystic Echinococcosis (CE) due to E. granulosus sensu lato is estimated at US\$ 2.1 billion annually ${ }^{15}$, and it is estimated to impose more than US\$230 million per year in $\operatorname{Iran}^{16}$. Therefore, control programs for zoonotic cestode infections are recommended by the World Health Organization ${ }^{17}$.

CE surveillance and control require persistent monitoring of infection in both definitive and intermediate hosts. Reliable diagnosis of taeniid infection in canine definitive hosts is essential for implementation of successful control programs. The eggs of different taeniid species are microscopically indistinguishable and this presents a major challenge in providing accurate data on the transmission patterns of each individual taeniid species. Several methods have been developed to differentiate taeniid species in dogs including antigenbased as well as DNA-based techniques. Copro-antigen ELISA and copro-DNA PCR have been successfully used for diagnosis of E. granulosus infection in canine definitive hosts ${ }^{18,19}$. However, these techniques are only able to discriminate $E$. granulosus from other taeniid species in dogs. It is documented that the biotic potential of E. granulosus, T. hydatigena and T. ovis are well-correlated well correlated, so differential diagnosis of these species in the same host could provide important information for selecting appropriate control strategies.

A multiplex PCR system has been developed by Trachsel et al..$^{20}$ using five primer pairs amplifying parts of nadl and small subunit of rRNA genes. Al-Sabi and Kapel demonstrated a multiplex PCR system for differentiating major Taenia species of rodents and carnivores ${ }^{21}$. However, multiplex PCR requires complex optimizations according to different sources of specimens. Therefore, simple, cost effective and efficient methods are needed to distinguish major Taeniidae species in dogs.
Microsatellites known as simple sequence repeats (SSR) or short tandem repeats (STR) are abundant unique tandem repeats of short (2-6 bp) DNA motifs in eukaryotic genomes which are usually polymorphic in number, because of their high abundance, widespread distribution in the genomes of various organisms ${ }^{22}$. The use of highly variable regions, such as microsatellites, provides more information about the parasite and its spatial and temporal distribution across different geographical locations. Microsatellites have proven to be a useful target for detection and identification of different Echinococcus species ${ }^{23}$. Recently, microsatellite analysis has been competently applied for investigating origin of E. multilocularis infection in intermediate hosts in a French wildlife park ${ }^{24}$.

Tandem repeats are distributed across eukaryote genome and they have been frequently found in rDNA regions including Internal Transcribed Spacers ${ }^{25,26}$. Internal transcribed spacers (ITS) and mitochondrial genes have been widely used in taxonomy and molecular phylogeny because it is easy to amplify even from small quantities and demonstrate a high degree of variation even between closely related species.

Simple and reliable identification of microsatellites in taeniid species of dogs could provide valuable information on the dynamics of transmission in endemic areas. The purpose of the present study was to identify microsatellite loci in mitochondrial as well as rDNA regions and to develop a simple reliable method for rapid differentiation of three Taenia species as well as E. granulosus sensu stricto in a single PCR system.

\section{MATERIALS AND METHODS}

Twenty isolates of each of three Taenia species, T. hydatigena, T. multiceps and T. ovis and 5 isolates of E. granulosus sensu stricto were collected from sheep during routine veterinary inspection from Tehran, Alborz and Kerman provinces. The parasites have already been sequenced and genetically characterized at the mitochondrial cytochrome $\mathrm{C}$ oxidase subunit $1(\mathrm{CO} 1)^{2,27,28}$. Sequence data were deposited in GenBank with the following accession numbers: T. hydatigena (JQ710588), T. multiceps (JQ710577) and T. ovis (JX134111) and E. granulosus sensu stricto (KF443137).

We found few genomic nucleotide records of taeniidae species in NCBI database. To predict any potential microsatellite regions in coding and non-coding regions, the complete mitochondrial sequence records of the four taeniids from NCBI Refseq were downloaded and analyzed by ChloroMitoSSRDB $2.0^{29}$, a microsatellitespecific software for organelle genomes. The screening was 
conducted on the basis of the following parameters: genome category (mitochondrial), repeat type (perfect/imperfect), and repeat size (mono to hexa).

On the other hand, the longest submitted sequences for rDNA as a universal genomic marker for species identification were collected according to the NCBI Nucleotides database. To predict potential microsatellite regions, we used GMATo v1.2. faster and accurate microsatellite mining at any length.

We have designed three primer sets (position 1-223, position 500-700 and position 700-1008) by Primer3 program (http://bioinfo.ut.ee/primer3-0.4.0/primer3/) based on the alignments of conserved flanking regions of tandem repeat sequences of rDNA for discrimination of taeniid species (Table 1). No rDNA sequence data were available for T. ovis.

According to the primer pair and the amplified fragment, PCR conditions were optimized as shown in Table 1 and the resulting products were analyzed by $2 \%$ agarose gel electrophoresis and visualized by using a UV transilluminator. PCR products of four representative samples were sequenced. The sequence data were aligned and edited using the BioEdit software ${ }^{31}$. All data were deposited in GenBank under the accession numbers KU639648 to KU639651. Regarding the high frequency of E. granulosus-T. hydatigena co-infection in dogs, a mixed sample containing $T$. hydatigena and $E$. granulosus sensu stricto was examined by PCR amplification.

\section{RESULTS}

Among the designed primer sets, the primer set1 (MF-1 and MR-233) provided the most discriminatory results in terms of the quality of amplification and banding profiles. The band sizes ranged from 210 to $312 \mathrm{bp}$ (Figure 1). Each taeniid species displayed a different band size that can be easily differentiated from other species. PCR amplification of mixed E. granulosus and T. hydatigena samples provided a mixed banding pattern (Figure 1, Lane E).
Motif types and repeat numbers were comparatively analyzed within the amplified fragment (data not shown). Different microsatellites found in ITS1 and whole mitochondrial regions are summarized in Table 2. The number of microsatellites varied from 2-19 and 1-4 in ITS1 and mitochondrial genome of taeniid species respectively. The highest number of perfect microsatellite motifs in ITS1 regions belonged to T. hydatigena and T. multiceps. Respectively, 71 and 11 perfect microsatellite loci in ITS1 and mitochondrial regions were found in the taeniid species. Among different types of tandem repeats, hexa motifs were significantly absent in ITS1 region while $41 \mathrm{Di}$ and $21 \mathrm{Tri}$ motifs were found in the taeniids. The majority of the motifs in the mitochondrial genome were Tetra (89) and Tri (70).

\section{DISCUSSION}

Molecular techniques provide us with very sensitive tools for differentiation of dog taeniids. Finding a primer site on the genome of major taeniid species co-existed in canids allows identification of genetic links between different isolates and the evaluation of parasite control by identifying the source of infection and the extension routes of the parasite across a specific region. Among several types of markers, common tandem repeat fragments within ITS1-rDNA region is considered especially useful for fingerprinting and transmission tracking due to the highly variant nature of the fragments ${ }^{32}$. Microsatellites within ITS1-rDNA are easy to amplify even from small specimen quantities and has a high degree of variation even between closely related species. This presents an additional advantage for using this primer set in field epidemiological studies of taeniids.

This study presents a conventional PCR primer set for differentiating different species of taeniids in dogs with a relatively rich content of variable microsatellites for fingerprinting and tracking transmission. The selected primer sets were proved to have more discriminatory power

Table 1 - Three primer sets designed by Primer Premier 5.0 software based on the alignments of conserved flanking regions of tandem repeat sequences for taeniid tapeworms (PCR conditions for all experiments were as follow: one cycle of $94{ }^{\circ} \mathrm{C} / 120 \mathrm{~s}$, 35 cycles of $94^{\circ} \mathrm{C} / 30 \mathrm{~s}, 59^{\circ} \mathrm{C} / 45 \mathrm{~s}$ and $72{ }^{\circ} \mathrm{C} / 45 \mathrm{~s}$, and a final extension of $72{ }^{\circ} \mathrm{C} / 180 \mathrm{~s}$

\begin{tabular}{|c|c|c|c|c|c|}
\hline & Primer name & Primer Sequence & Length & Annealing Temp. & Product $(\mathrm{bp})^{\star \star}$ \\
\hline \multirow{2}{*}{ Set 1} & $\mathrm{MF}-1$ & 5' GTCGTAACAAGGTTTCCGTAGGTG 3' & 24 & \multirow{2}{*}{$52-61(59)^{*}$} & \multirow{2}{*}{223} \\
\hline & MR-233 & 5' AAGGCTAGAGGCAGACTAGGCAC 3' & 23 & & \\
\hline \multirow{2}{*}{ Set 2} & MF-509 & 5' CGACAGTAGCGATGACRTTGAGG 3' & 23 & \multirow{2}{*}{$52-61(59)^{*}$} & \multirow{2}{*}{220} \\
\hline & MR-728 & 5' ACGCACGAGCCRAGTGATCCACC 3' & 23 & & \\
\hline \multirow{2}{*}{ Set 3} & MF-766 & 5' ATCGCAGACTGCTTTGAACATCG 3' & 23 & \multirow{2}{*}{$52-61(59)^{*}$} & \multirow{2}{*}{343} \\
\hline & MR-1108 & 5' GTGAACTGTGACTGCACGACCA 3' & 23 & & \\
\hline
\end{tabular}

* Optimum temperature; ** Fragment size for Taenia hydatigena 

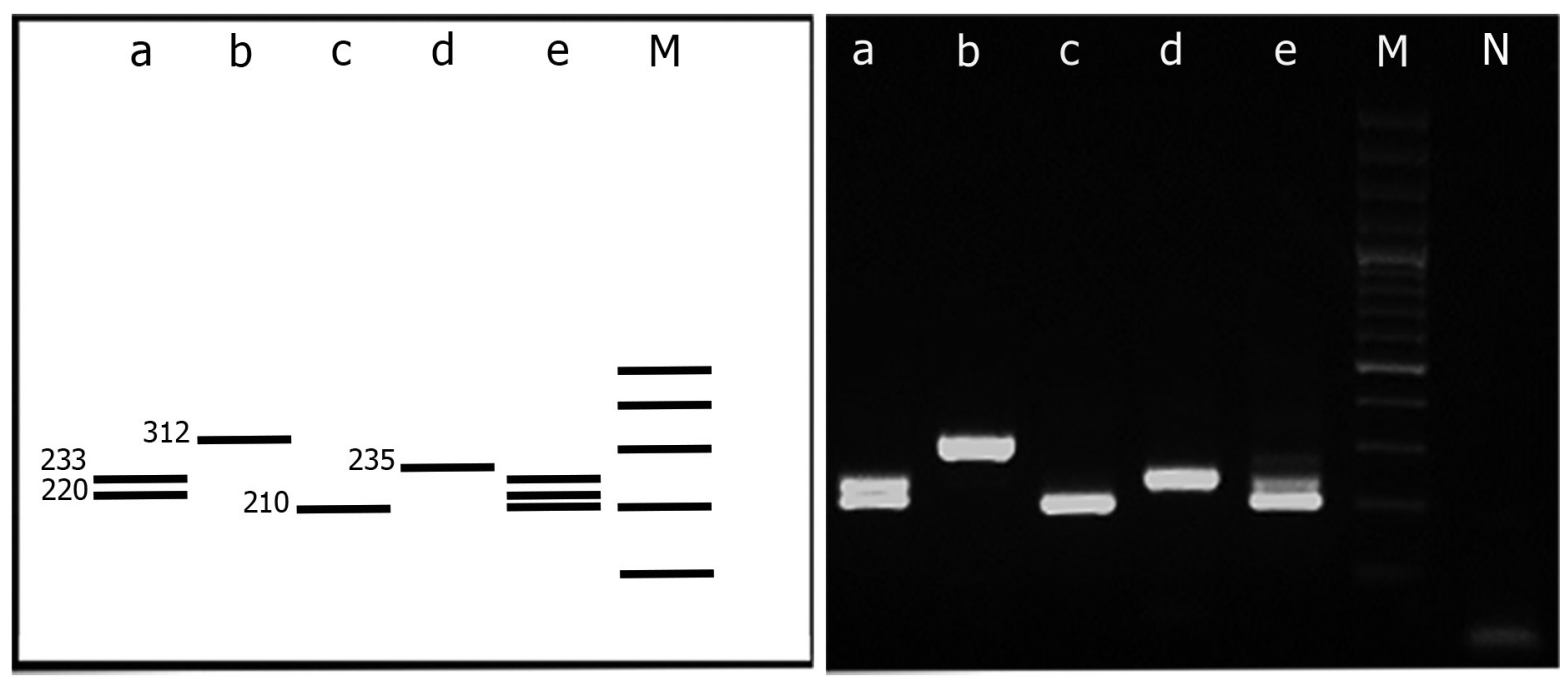

Figure 1 - Agarose gel electrophoresis showing different banding patterns of four taeniid tapeworms using rDNA-ITS-1 microsatellitedriven primer pair MF-1/MR-233. A) Taenia hydatigena; B) T. ovis; C) Echinococcus granulosus sensu stricto; D) T. multiceps; E) E. granulosus + T. hydatigena. M. 100-bp DNA size marker, N. negative control.

for the differentiation of the common taeniid species of dogs. The PCR profile for each individual taeniid was species-specific and a well-defined pattern was observed in the PCR amplification of mixed E. granulosus-T. hydatigena DNA templates.

Our knowledge on the frequency and distribution of microsatellites in Taeniidae species is limited. In the screening phase of the study we tried to find potential loci of STR/SSR in ITS1 as well as mitochondrial regions via computational prediction. Findings of the present study revealed 71 and 11 microsatellite loci in ITS1 and mitochondrial regions, respectively. As shown in Table 2, maximum number of microsatellites in ITS1 region was found in T. hydatigena and T. multiceps followed

Table 2 - Characteristics of ITS1 and mitochondrial microsatellites found in 7 taeniid species

\begin{tabular}{|c|c|c|c|c|c|c|c|}
\hline Region & Organism & Accession № & $\begin{array}{l}\text { Length } \\
\text { (bp) }\end{array}$ & Repetition & $\begin{array}{c}\text { № of } \\
\text { perfect } \\
\text { sites }^{*}\end{array}$ & $\begin{array}{c}\text { № of } \\
\text { imperfect } \\
\text { sites* }^{*}\end{array}$ & Type of tandem repeat \\
\hline \multirow{7}{*}{ Mitochondrial } & T. hydatigena & NC012896 & 13,492 & 3 to 6 & 0 & 15 & 3 Di, 3 Tri, 7 Tetra, 1 Penta, 1 Hexa \\
\hline & T. multiceps & NC012894 & 13,693 & 3 to 9 & 1 & 28 & 7 Di, 8 Tri, 9 Tetra, 3 Penta, 2 Hexa \\
\hline & T. saginata & NC009938 & 13,670 & 3 to 7 & 4 & 35 & $\begin{array}{c}8 \text { Di, } 13 \text { Tri, } 14 \text { Tetra, } 2 \text { Penta, } \\
2 \text { Hexa }\end{array}$ \\
\hline & T. ovis & NC020153 & 13,536 & 3 to 7 & 3 & 30 & 7 Di, 6 Tri, 14 Tetra, 5 Penta, 1 Hexa \\
\hline & T. solium & NC004022 & 13,709 & 3 to 7 & 2 & 29 & $\begin{array}{c}3 \text { Di, } 10 \text { Tri, } 15 \text { Tetra, } 2 \text { Penta, } \\
1 \text { Hexa }\end{array}$ \\
\hline & E. granulosus & NC008075 & 13,588 & 3 to 6 & 0 & 32 & 3 Di, 15 Tri, 12 Tetra, 2 Penta \\
\hline & E. multilocularis & NC000928 & 13,738 & 3 to 7 & 1 & 39 & $\begin{array}{c}4 \text { Di, } 15 \text { Tri, } 18 \text { Tetra,1 Penta, } \\
2 \text { Hexa }\end{array}$ \\
\hline \multirow{7}{*}{ rDNA-ITS1 } & T. hydatigena & FJ886757 & 1,259 & 5 to 14 & 19 & $N D^{\star \star}$ & 12 Di, 6 Tri, 1 Tetra \\
\hline & T. multiceps & FJ886762 & 1,359 & 3 to13 & 19 & ND & 10 Di, 5 Tri, 2 Tetra, 2 Penta \\
\hline & T. saginata & AY392045 & 782 & 3 t0 4 & 10 & ND & 7 Di, 2 Tri, 1 Tetra \\
\hline & T. ovis & KU639651 & 312 & 3 & 2 & ND & 2 Tri \\
\hline & T. solium & AF372565 & 744 & 5 to 10 & 2 & ND & $1 \mathrm{Di}, 1$ Tetra \\
\hline & E. granulosus & AY969043 & 1,046 & 3 to 5 & 11 & ND & $7 \mathrm{Di}, 4 \mathrm{Tri}$ \\
\hline & E. multilocularis & AJ237778 & 996 & 3 to 6 & 8 & ND & 4 Di, 2 Tri, 2 Tetra \\
\hline
\end{tabular}

*Single repeats motifs were not calculated in motifs separation. ${ }^{* *}$ Not Determined 
by $T$. saginata and E. granulosus sensu stricto. Taenia hydatigena has previously been shown to have a high degree of variability in different regions of the world 33,34 probably because of the highest prevalence and intensity of T. hydatigena transmission among the four taeniid species in this study ${ }^{5-7}$.

This study shows rich microsatellite content in the parasite ITS1 region compared to other taeniid species. In the present study, a higher number of perfect microsatellite motifs was found in ITS1 than in the mitochondrial region. This is in agreement with the fact that the coding regions have less frequent microsatellites than the non-coding ones ${ }^{22}$. In addition, ITS1 dinucleotide motifs were the most abundant in taeniids as previously shown in other species ${ }^{35}$.

Microsatellites are popular genetic markers and very useful tools for molecular epidemiological studies. In addition, microsatellite markers have been successfully used for fingerprinting and tracking transmission of echinococcosis ${ }^{24}$. In the first, tandem repeats detected in $223 \mathrm{bp}$ amplified fragment of $T$. hydatigena (position 50), the number of repetitions was significantly variable (5-7 repeats) among different isolates from China (FJ886758FJ886761) ${ }^{36}$. Insertions/deletions could potentially change the motif types and repeats within a microsatellite fragment. Five repeats of GCT in three Chinese isolates of T. hydatigena has been replaced by 4 repeats of TGC in one isolate from the same area. Comparing 5 isolates of $E$. granulosus from Poland, Iran and India revealed that a tetra motif, TGGT is present in the Indian and Iranian isolates while the motif is notably absent in the Polish isolates (AY969043, DQ011674, KJ363926, AJ245928, AJ245930). These phenomena could potentially enable us to precisely identify possible genetic links between different isolates of the same tapeworm species in a defined geographical region.

We found high AT content in mitochondrial microsatellites compared to nuclear regions. Similar findings have been published for mitochondrial microsatellites in parasites as well as free-living nematodes. Accordingly, in ITS1 region, a higher GT content was found in the study in agreement with Pajuelo et al. ${ }^{35}$.

This study described microsatellite markers in major taeniid species of medical and veterinary importance. Very little information has been available on this issue and this study presents the frequency and distribution of potential microsatellite markers in common Taeniidae of dogs. The primers designed based on ITS1 microsatellites were proved to be a potential tool for tracking transmission and epidemiological studies on taeniid infections particularly cystic echinococcosis. Several epidemiological studies are required for evaluating the reliability and sensitivity of the tool in field conditions.

\section{ACKNOWLEDGEMENTS}

The authors would like to thank Mr. Hossein Kamyabi for his kind cooperation in collecting parasite materials. This study was financially supported by the Vice-Chancellor for Research and Technology, Kerman University of Medical Sciences, grant No 93-232.

\section{REFERENCES}

1. Deplazes P, Rinaldi L, Alvarez Rojas CA, Torgerson PR, Harandi MF, Romig T, et al. Global distribution of alveolar and cystic echinococcosis. Adv Parasitol. 2017;95:315-493.

2. Rostami S, Salavati R, Beech RN, Babaei Z, Sharbatkhori M, Baneshi MR, et al. Molecular and morphological characterization of the tapeworm Taenia hydatigena (Pallas, 1766) in sheep from Iran. J Helminthol. 2015;89:150-7.

3. Wondimu A, Abera D, Hailu Y. A study on the prevalence, distribution and economic importance of Cysticercus tenuicollis in visceral organs of small ruminants slaughtered at an abattoir in Ethiopia. J Vet Med Anim Health. 2011;3:67-74.

4. Mirbadie SR, Kamyabi H, Mohammadi MA, Shamsaddini S, Harandi MF. Copro-PCR prevalence of Echinococcus granulosus infection in dogs in Kerman, south-eastern Iran. J Helminthol. In Press 2017.

5. Dalimi A, Sattari A, Motamedi G. A study on intestinal helminthes of dogs, foxes and jackals in the western part of Iran. Vet Parasitol. 2006;142:129-33.

6. Radfar MH, Tajalli S, Jalalzadeh M. Prevalence and morphological characterization of Cysticercus tenuicollis (Taenia hydatigena cysticerci) from sheep and goats in Iran. Veterinarski Arhiv. 2005;75:469-76.

7. Hosseini SH, Habibi M. Gastrointestinal helminthes of sheepdog in Ardestan (Isfahan province-Iran). Pajouhesh-va-Sazandegi. 2000;13:108-9.

8. Achenef M, Markos T, Feseha G, Hibret A, Tembely S. Coenurus cerebralis infection in Ethiopian highland sheep: incidence and observations on pathogenesis and clinical signs. Trop Anim Health Prod. 1999;31:15-24.

9. Rostami S, Beech RN, Salavati R, Baneshi MR, Kamyabi H, Harandi MF. Morphometric analysis of larval rostellar hooks in Taenia multiceps of sheep in Iran and its association with mitochondrial gene variability. Iran J Parasitol. 2013;8:579-85.

10. Pestechian N, Rasouli A, Yoosefi HA. Distribution of intestinal worms among stray dogs in Isfahan, Iran. J Isfahan Med Sch. 2012;29:1-7.

11. Shi W, He W, Guo X, Liu Q, Gao S, Zhan F, et al. The first outbreak of Taenia ovis infection in China. Parasitol Int. 2016;65:422-3

12. Federer K, Armua-Fernandez MT, Gori F, Hoby S, Wenker C, Deplazes P. Detection of taeniid (Taenia spp., Echinococcus spp.) eggs contaminating vegetables and fruits sold in European 
markets and the risk for metacestode infections in captive primates. Int J Parasitol Parasites Wildl. 2016;5:249-53.

13. Scala A, Pipia AP, Dore F, Sanna G, Tamponi C, Marrosu R, et al. Epidemiological updates and economic losses due to Taenia hydatigena in sheep from Sardinia, Italy. Parasitol Res. 2015;114:3137-43

14. Oryan A, Moghaddar N, Gaur SN. Metacestodes of sheep with special reference to their epidemiological status, pathogenesis and economic implications in Fars Province, Iran. Vet Parasitol. 1994;51:231-40.

15. Budke CM, Deplazes P, Torgerson PR. Global socioeconomic impact of cystic echinococcosis. Emerg Infect Dis. 2006;12:296-303.

16. Harandi MF, Budke CM, Rostami S. The monetary burden of cystic echinococcosis in Iran. PLoS Negl Trop Dis. 2012;6:e1915.

17. World Health Organization. Department of Control of Neglected Tropical Diseases. Sustaining the drive to overcome the global impact of neglected tropical diseases: second WHO report on neglected tropical diseases. Geneva: WHO; 2013.

18. Abbasi I, Branzburg A, Campos-Ponce M, Abdel Hafez SK, Raoul F, Craig PS, et al. Copro-diagnosis of Echinococcus granulosus infection in dogs by amplification of a newly identified repeated DNA sequence. Am J Trop Med Hyg. 2003; 69:324-30.

19. Morel N, Lassabe G, Elola S, Bondad M, Herrera S, Marí C, et al. A monoclonal antibody-based copro-ELISA kit for canine echinococcosis to support the PAHO effort for hydatid disease control in South America. PLoS Negl Trop Dis. 2013;7;e1967.

20. Trachsel D, Deplazes P, Mathis A. Identification of taeniid eggs in the faeces from carnivores based on multiplex PCR using targets in mitochondrial DNA. Parasitology. 2007;134:911-20.

21. Al-Sabi MN, Kapel CM. Multiplex PCR identification of Taenia spp. in rodents and carnivores. Parasitol Res. 2011;109:1293-8.

22. Oliveira EJ, Pádua JG, Zucchi MI, Vencovsky R, Vieira ML. Origin, evolution and genome distribution of microsatellites. Genet Mol Biol. 2006;29:294-307.

23. McManus DP, Thompson RC. Molecular epidemiology of cystic echinococcosis. Parasitology. 2003;127 Suppl:S37-51.

24. Umhang G, Lahoreau J, Hormaz V, Boucher JM, Guenon A, Montange D, et al. Surveillance and management of echinococcus multilocularis in a wildlife park. Parasitol Int. 2016; 65:245-50.

25. Lunt DH, Whipple LE, Hyman BC. Mitochondrial DNA variable number tandem repeats (VNTRs): utility and problems in molecular ecology. Mol Ecol. 1998;7:1441-55.
26. Richard GF, Kerrest A, Dujon B. Comparative genomics and molecular dynamics of DNA repeats in eukaryotes. Microbiol Mol Biol Rev. 2008;72:686-727.

27. Rostami S, Salavati R, Beech RN, Sharbatkhori M, Babaei $\mathrm{Z}$, Saedi S, et al. Cytochrome c oxidase subunit 1 and $12 \mathrm{~S}$ ribosomal RNA characterization of Coenurus cerebralis from sheep in Iran. Vet Parasitol. 2013;197:141-51.

28. Rostami S, Talebi S, Babaei Z, Sharbatkhori M, Ziaali N, Rostami H, et al. High resolution melting technique for molecular epidemiological studies of cystic echinococcosis: differentiating G1, G3, and G6 genotypes of Echinococcus granulosussensu lato. Parasitol Res. 2013;112:3441-7.

29. Sablok G, Padma Raju G, Mudunuri SB, Prabha R, Singh DP, Baev V, et al. ChloroMitoSSRDB 2.00: more genomes, more repeats, unifying SSRs search patterns and on-the-fly repeat detection. Database (Oxford). 2015;2015:bav084.

30. Wang X, Lu P, Luo Z. GMATo: a novel tool for the identification and analysis of microsatellites in large genomes. Bioinformation. 2013;9:541-4.

31. Hall T. BioEdit, version 5.0.9. Raleigh: North Carolina State University; 1999.

32. Lymbery AJ, Thompson RC. The molecular epidemiology of parasite infections: tools and applications. Mol Biochem Parasitol. 2012;181:102-16.

33. Boufana B, Scala A, Lahmar S, Pointing S, Craig PS, Dessì G, et al. A preliminary investigation into the genetic variation and population structure of Taenia hydatigena from Sardinia, Italy. Vet Parasitol. 2015;214:67-74.

34. Rostami S, Shariat Torbaghan S, Dabiri S, Babaei Z, Ali Mohammadi M, Sharbatkhori M, et al. Genetic characterization of Echinococcus granulosus from a large number of formalinfixed, paraffin-embedded tissue samples of human isolates in Iran. Am J Trop Med Hyg. 2015;92:588-94.

35. Pajuelo MJ, Eguiluz M, Dahlstrom E, Requena D, Guzmán F, Ramirez M, et al. Identification and characterization of microsatellite markers derived from the whole genome analysis of Taenia solium. PLoS Negl Trop Dis. 2015;9:e0004316.

36. Dai RS, Liu GH, Song HQ, Lin RQ, Yuan ZG, Li MW, et al. Sequence variability in two mitochondrial DNA regions and internal transcribed spacer among three cestodes infecting animals and humans from China. J Helminthol. 2012;86:24551. 